\title{
Reflective function and secure attachment as resilient strategies in impaired adolescents
}

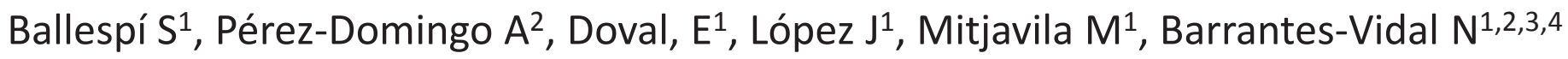

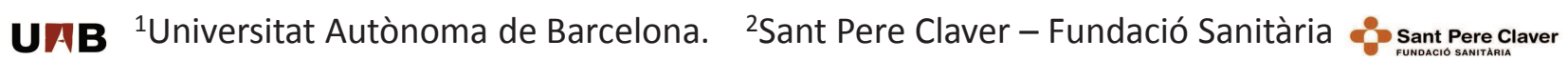 \\ cibersam ${ }^{3}$ Instituto de Salud Carlos III, CIBERSAM ${ }^{4}$ University of North Carolina at Greensboro
}

\section{INTRODUCTION}

In a difficult transition such as adolescence resilient areas of personality will be valuable to prevent suffering and impairment. Resilient areas are those processes identified as helpful to overcome adversity (Ungar, 2012). Among all possible resilient factors in the adolescence attachment style and reflective function (RF) are underrepresented in the scientific literature. Secure attachment style is a resilient process because it leads to approach others with confidence and facilitates relationships (Flaherty, \& Sadler, 2011). For people with a secure attachment style it is easy to become emotionally close to others. They are comfortable depending on others and having others depending on them, and they do not worry about being alone (Bartholomew, \& Horowitz, 1991).

By contrast, Fearful-Avoidant attachment style is a kind of insecure attachment based on mixed feelings implying mistrust and fear of others and it is associated to risk for anxious disorders (Eng, Heimberg, Hart, Schneier, \& Liebowitz, 2001). People with Fearful-Avoidant attachment style feel uncomfortable getting close to others. They want emotionally close relationships but they find it difficult to trust others or to depend on them because they worry that they will be hurt if they allow themselves to become too close to others (Bartholomew, \& Horowitz, 1991).

Furthermore, the attachment style is related to reflective function (RF), i.e. the capacity to interpret human behavior in terms of mental states (Fonagy, \& Target, 2006). By attributing mental states (such as feelings, desires or intentions) to others people make human behavior meaningful and predictable. It is well-known that a deficit in RF (also called mentalization) is also associated to psychopathology (Bevington, Fuggle, Fonagy et al., 2013).

Table 1. Linear Regressions of Social Anxiety. Whole Sample $(n=276)$

\begin{tabular}{|c|c|c|c|c|c|c|c|c|c|}
\hline Criterion & $\#$ & Predictor & Step $1^{\circ}$ & Step 2 & Step 3 & $\mathrm{R}^{2}$ & F(Model) & $\Delta R^{2}$ & $\Delta \mathrm{F}$ \\
\hline Social Anxiety & 1 & Resiliency $y^{1}$ & $-31^{* * *}$ & $-.20 \cdots$ & $-20^{* *}$ & .10 & $29.04^{* * *}$ & & \\
\hline \multirow[t]{3}{*}{ (SAS-A) } & 2 & Mentalization & & $.49^{\cdots \cdots}$ & $.47^{* * *}$ & .32 & $65.02^{* * *}-1$ & .23 & $91.38^{* * *}$ \\
\hline & 3 & Secure Attachment & & & -.05 & .33 & $33.32^{* * *}$ & .01 & 1.42 \\
\hline & & Fearful-Avoid. Attach. & & & .06 & & & & \\
\hline
\end{tabular}

Table 2. Linear Regressions of Measures of Impairment. Subsample ( $n=80)$

\begin{tabular}{|c|c|c|c|c|c|c|c|c|c|}
\hline Criterion & $\#$ & Predictor & Step $1^{\circ}$ & Step 2 & Step 3 & $\mathrm{R}^{2}$ & F(Model) & $\Delta R^{2}$ & $\Delta F$ \\
\hline \multirow{4}{*}{ (SAS-A) } & 1 & Resiliency $^{1}$ & $-.31^{\cdots \cdots}$ & $-.20^{* * *}$ & $-.18^{* \cdots}$ & .10 & $29.12^{\cdots * *}$ & & \\
\hline & 2 & Mentalization & & $.49^{\cdots *}$ & $.45 * \cdots$ & .32 & $64.75^{\cdots *}$ & .23 & $90.84 \cdots$ \\
\hline & 3 & Secure Attachment & & & $-.35^{*}$ & .36 & $37.73^{\cdots *}$ & .04 & $7.58^{\cdots \cdots}$ \\
\hline & & Fearful-Avoid. Attach. & & & $.44^{* \cdots}$ & & & & \\
\hline General Anxiety & 1 & Resiliency $^{1}$ & $-.26^{*}$ & -.17 & -.11 & .07 & $5.07^{*}$ & & \\
\hline \multirow[t]{3}{*}{ (MASC) } & 2 & Mentalization & & $.37^{* *}$ & .15 & .19 & $8.47^{\cdots *}$ & .13 & $11.16^{* \prime}$ \\
\hline & 3 & Secure Attachment & & & $-.24^{*}$ & .43 & $13.29^{\cdots *}$ & .24 & $14.86^{* *}$ \\
\hline & & Fearful-Avoid. Attach. & & & $.42^{* *}$ & & & & \\
\hline \multirow{4}{*}{ (SsQ) } & 1 & Resiliency $^{1}$ & .04 & .10 & .12 & .00 & .09 & & \\
\hline & 2 & Mentalization & & $.28^{*}$ & $.25^{\circ}$ & .08 & 3.01 & .08 & $5.93^{*}$ \\
\hline & 3 & Secure Attachment & & & .13 & .13 & $2.53^{*}$ & .05 & 1.97 \\
\hline & & Fearful-Avoid. Attach. & & & .23 & & & & \\
\hline Depression & 1 & Resiliency $^{2}$ & $-.36^{*}$ & $-.29^{*}$ & -.17 & .13 & $10.41^{*}$ & & \\
\hline \multirow[t]{3}{*}{ (BDI-II) } & 2 & Mentalization & & $.37^{*}$ & $.24^{*}$ & .26 & $12.44^{\cdots *}$ & .13 & $12.76^{\prime \prime}$ \\
\hline & 3 & Secure Attachment & & & -.04 & .39 & $10.80^{\cdots *}$ & .13 & $7.05^{* *}$ \\
\hline & & Fearful-Avoid. Attach. & & & $.39^{*}$ & & & & \\
\hline Self-Esteem & 1 & Resiliency $^{2}$ & $.35^{* \prime}$ & $.29^{*}$ & .16 & .13 & $10.27^{*}$ & & \\
\hline \multirow[t]{3}{*}{ (Rosenberg's Scale) } & 2 & Mentalization & & $-.37^{* *}$ & -.20 & .25 & $12.06^{* *}$ & .13 & $12.25^{* \prime}$ \\
\hline & 3 & Secure Attachment & & & .17 & .40 & $11.32^{\cdots *}$ & .14 & $8.15^{\prime \prime}$ \\
\hline & & Fearful-Avoid. Attach. & & & $-.36^{* *}$ & & & & \\
\hline Sociometric & 1 & Resiliency $^{2}$ & $.28^{*}$ & $.26^{*}$ & -.16 & .08 & $6.33^{*}$ & & \\
\hline \multirow[t]{3}{*}{ Status (BSQ) } & 2 & Mentalization & & -.12 & .04 & .09 & $3.69^{*}$ & .01 & 1.04 \\
\hline & 3 & Secure Attachment & & & $.26^{*}$ & .21 & $4.52^{* *}$ & .11 & $4.94^{*}$ \\
\hline & & Fearful-Avoid. Attach. & & & -.22 & & & & \\
\hline
\end{tabular}

References



\section{GOAL \& HYPOTHESES}

The aim of this study is to analyze to what extent attachment style and reflective function modulate resiliency in a sample of adolescents from the general population. We firstly hypothesize that good mentalization capacity and secure attachment style will protect adolescents from impairment. Given that most analyses are run with a subsample in which social-anxious participants are overrepresented, and given that social anxiety is related to the fearful-avoidant style, we also predict that fearful-avoidant attachment style will also modulate resiliency along with mentalization capacity.

\section{METHOD}

Participants. A sample of 276 adolescents aged 12 to 18 years old were assessed in a First Phase of a study about Social Anxiety. For the Second Phase, 80 adolescents were selected on the basis of their level of social anxiety. This subsample was formed by 40 participants with high social anxiety and 40 healthy controls with low social anxiety.

Instruments. Mentalization Questionnaire (MZQ; Hausberg et al., 2012), Connor-Davidson Resilience Scale - 10 Items version (CD-RISC-10; Connor, \& Davidson, 2003), Ego Resiliency Scale - Revised (ER89-R; Alessandri et al., 2012), Relationship Questionnaire (RQ) and Relationship Style Questionnaire (RSQ; Bartholomew, \& Horowitz, 1991), Social Anxiety Scale for Adolescents (SAS-A; La Greca, \& López, 1998), Multidimensional Anxiety Scale for Children (MASC; March et al., 1997), Beck Depression Inventory II (BDI-II; Beck, Steer, \& Brown, 1996), Rosenberg Self-Esteem Scale (RSES; Rosernberg, 1965), Somatic Symptoms Questionnaire (SSQ; Domènech-Llaberia et al., 2004), Brief Sociometric Scale (BSQ; Ballespí, 2013).

Procedure. Once we had received the informed consent all data were collected in the schools. Multivariate linear regression is used for modeling data.


RESULTS

In the whole sample $(\mathrm{N}=276)$ Social Anxiety (SAS-A) is predicted by resiliency and problems of mentalization but not by the attachment style. In the subsample $(n=80)$, resiliency towards impairment is modulated by mentalization and attachment style in most cases. As it is shown in Table 2, resiliency, mentalization, secure attachment and not having fearful attachment style protect from social anxiety (SAS-A). Resiliency is a protective factor from general anxiety (MASC) when entered at first step but no longer so when fearful avoidant attachment style is added to the equation. Impairment in mentalization accounts for variance in somatic complaints (SSQ) when no other predictors do. Regarding depression (BDI-II), resiliency protects from it until mentalization and attachment are added to the equation. Then, the effect of resiliency is mediated by deficit in mentalization and by not having a fearful avoidant attachment style, as it also happens with the scores from the Rosenberg's Self-Esteem Scale (RSES). Finally, also popularity and good acceptance among others (Sociometric Status) are predicted by the level of resiliency but one more time this effect is mediated by showing a secure attachment style.

\section{DISCUSSION \& CONCLUSION}

Results mostly support our predictions of the moderation effect of attachment style and mentalization on the resiliency in the adolescence. Social relationships are an important source of support against adversity in the adolescence. It is well-known that an adolescent with a good social framework is more likely to be resilient in the transition to the adulthood than adolescents with less social support or fewer close others (e.g. those avoiding interaction because of high levels of social anxiety). The current study provides evidence of secure attachment style and good mentalization capacity contributing to reduce impairment in adolescents with social anxiety. It should be taken into account that most of the current results have been obtained with a subsample of participants with high social anxiety. Thus, future investigations should evaluate whether mentalization and attachment style modulate resiliency also in a wide sample of general population. 